\section{South-East Scotland trabeculectomy survey}

P Cackett ${ }^{1}$, J Vallance ${ }^{2}$, C Cobb², H Devlin'1, A Simpson ${ }^{3}$ and R Sanders ${ }^{3}$
Eye (2007) 21, 46-51. doi:10.1038/sj.eye.6702118; published online 7 October 2005

Keywords: trabeculectomy; antimetabolite; intraocular pressure; trainee; consultant

\section{Introduction}

The National Survey of Trabeculectomy (NST) was carried out in 1996 in order to assess glaucoma surgical practice in the United Kingdom (UK). ${ }^{1-3}$ In total, 382 consultants (22.6\% of whom had a specialist interest in glaucoma) recruited four consecutive patients undergoing trabeculectomy. A wide variation in the operative technique was noted. The NST concluded that outcome and complications were unrelated to the consultant's special interest, level of activity, type of hospital, or region. The issue of surgical trainees performing glaucoma surgery was not addressed.

The NST is representative of glaucoma surgery in the UK nearly a decade ago. Since 1996 much has changed in the practice of glaucoma in the UK. The medical treatment of glaucoma has seen a steady increase in the use of new and powerful intraocular pressure (IOP)-reducing topical agents. ${ }^{4}$ As a result, over the same period there has been a steady decline in the number of trabeculectomies being performed. ${ }^{4,5}$ This is occurring during a time when higher surgical trainees (HST) are expected to perform a set number of glaucoma procedures. ${ }^{6}$ The use of antimetabolites intraand postoperatively has increased considerably since 1996 with more aggressive postoperative management of the failed trabeculectomy bleb. ${ }^{7}$

In South-East Scotland a specialist glaucoma service has been established since January 1999, where two glaucoma consultants are responsible for performing over $90 \%$ of all glaucoma surgery. With the setting up of this new service, taking place in a background of changes in the management of glaucoma, we wanted to examine our own cohort of surgical
There are no proprietary interests with this paper
Received: 24 March 2005 Accepted in revised form: 19 August 2005

Published online: 7 October 2005
${ }^{1}$ Princess Alexandra Eye Pavilion, Chalmers Street,

${ }^{2}$ Ninewells Hospital, Princess Alexandra Eye Edinburgh EH3 9HA Scotland Fax: + 441315361574 E-mail: pete@ (n)

\begin{abstract}
Conclusion Our study highlights significant changes in the practice and outcome of trabeculectomy compared to the national survey conducted a decade ago.

Abstract

Aim Assessment of trabeculectomy in South-East Scotland (SESTS) with comparison and outcon surger $(P<0.001)$, and a higher rate of early complications $(P<0.025)$. There was no significant difference in outcome at intraocular pressure (IOP) $<2 / 3$ listing IOP (qualified and unqualified) between the SESTS and the NST. unqualified success of IOP $<21 \mathrm{mmHg}$ IOP $<16 \mathrm{mmHg}$ in the SESTS than the NST greater than one Snellen line was more common in the NST $(P<0.01)$ as was the use cases returned to theatre more frequently $(P<0.025)$ and merited more bleb intervention $(P<0.01)$ than consultant cases, but the longterm outcome was similar.
\end{abstract}

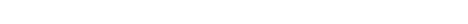


patients. We therefore carried out a survey of our surgical practice in the South-East Scotland trabeculectomy survey (SESTS). The data from this survey were used to compare with the NST and aid the documentation of the changes seen in the practice of trabeculectomy since 1996. Also, because of the demands on surgical training, we wanted to compare the outcomes of the glaucoma consultants and the HST.

\section{Methods}

A retrospective study of consecutive primary trabeculectomies performed at two centres in SE Scotland (Edinburgh and Dunfermline) was carried out. All trabeculectomies surveyed were performed by one of two glaucoma consultants or an HST under close scrubbed supervision of a glaucoma consultant. The trabeculectomies were performed between January 1996 and June 2003 in Dunfermline, and between January 2001 and June 2003 in Edinburgh. The differing start dates at these two centres coincided with the appointments of new glaucoma consultants to these units. The retrieval rates for case notes were $91 \%$ for Edinburgh and $92 \%$ for Dunfermline. Patients who had previously undergone glaucoma surgery were excluded from the study. Advanced visual field loss was defined as a field defect within five degrees of fixation. ${ }^{8}$ The minimum length of follow-up after trabeculectomy was 1 year.

Both glaucoma consultants performed trabeculectomy with a fornix-based conjunctival flap, triangular or rectangular scleral flap, punch sclerostomy, peripheral iridectomy, and fixed sutures to the scleral flap and conjunctiva. The tension of the sutures was adjusted based on the depth of the anterior chamber and patency of the fistula. Antimetabolites were used based on risk factors for failure. ${ }^{9}$ Five-fluorouracil (5-FU) was applied at a concentration of $25 \mathrm{mg} / \mathrm{ml}$ for $5 \mathrm{~min}$ and mitomycinc (MMC) at a concentration of 0.2 or $0.4 \mathrm{mg} / \mathrm{ml}$ for $2 \mathrm{~min}$. All patients were prescribed postoperative topical steroids for a minimum of 3 months and had stringent follow-up postoperatively on days 1, 7, 14 and months 1 , 2,3 . Hypotony was defined as IOP $<7 \mathrm{mmHg}$ at the first postoperative visit, excluding the first postoperative day.
All bleb interventions and postoperative interventions were carried out by the glaucoma consultant or HST under close supervision of the glaucoma consultant.

The outcome measures of success for the SESTS were identical to the NST so that direct comparisons could be made. ${ }^{2}$ Main outcome measure of success was defined as final IOP less than two-thirds the preoperative IOP at 1 year following trabeculectomy. Secondary outcome measures of success were final IOP $<21 \mathrm{mmHg}$ and final IOP $<16 \mathrm{mmHg}$. These were further defined as unqualified (excluding patients on anti-glaucoma medications) or qualified (including patients on antiglaucoma medications).

All information was collected on scanning laser forms and manually validated. The information was then transported to Microsoft Excel for statistical analysis. $\chi^{2}$ statistical tests were used for categorical variables. Student's $t$-test or Mann-Whitney $U$ test as appropriate were used for continuous variables.

\section{Results}

In all, 304 patients records were audited, 124 from Edinburgh and 180 from Dunfermline. The mean length of follow-up after surgery was 2.74 years (standard deviation (SD) 1.73).

The mean age of patients was 66.8 years (SD 13.4) (vs 69.2 years (SD 10.9) in NST); 151 (49.7\%) were men (vs $51.7 \%$ in NST) with $99.7 \%$ being Caucasian. The underlying diagnosis was primary open angle glaucoma (POAG) in 65.5\%, pseudoexfoliation (PXF) in 6.9\%, normal tension glaucoma (NTG) in $7.6 \%$, pigment dispersion syndrome (PDS) in $1.6 \%$, and other complicated glaucoma in $18.4 \%$. The mean IOP at diagnosis was $29.4 \mathrm{mmHg}$ (SD 10.4) (vs $29.2 \mathrm{mmHg}$ (SD 7.5) in NST), the highest preoperative IOP was $34.3 \mathrm{mmHg}$ (SD 10.2) and at listing was $24.7 \mathrm{mmHg}$ (SD 7.7) (vs $26.4 \mathrm{mmHg}$ (SD 5.9) in NST) for the whole cohort, but differed between the diagnostic categories (Table 1). The patients in the SESTS were significantly younger than in the NST $(P<0.01)$ but the sex distribution was similar between the two studies. The IOP at diagnosis was similar between the two studies

Table 1 Age, presenting and listing IOPs according to diagnostic categories

\begin{tabular}{lcccccccr}
\hline Diagnosis & $\begin{array}{c}\text { Number of } \\
\text { patients }\end{array}$ & $\%$ & $\begin{array}{c}\text { Mean age } \\
\text { (years) }\end{array}$ & $95 \%$ CI & $\begin{array}{c}\text { Mean IOP at } \\
\text { diagnosis (mmHg) }\end{array}$ & $95 \%$ CI & $\begin{array}{c}\text { Mean listing } \\
\text { IOP (mmHg) }\end{array}$ & $\begin{array}{c}95 \% \text { CI } \\
\text { COAG }\end{array}$ \\
\hline PXF & 199 & 65.5 & 68.9 & $67.4-70.4$ & 29.3 & $28.2-30.4$ & 23.8 & $22.8-24.7$ \\
NTG & 21 & 6.9 & 71.6 & $68.2-74.9$ & 34.6 & $28.3-40.9$ & 26.6 & $23.6-29.7$ \\
Pigment & 5 & 7.6 & 72.8 & $68.9-76.7$ & 20.5 & $18.3-22.8$ & 18.4 & $17.2-19.6$ \\
Other & 5 & 1.6 & 59.8 & $44.9-74.7$ & 28 & $15.9-40.1$ & 20 & $17.8-22.2$ \\
\hline
\end{tabular}


but the listing IOP was significantly lower in the SESTS $(P<0.001)$.

Patients were on medication for an average of 5.35 years prior to surgery (range $0.3-25.8$ years). The mean number of topical medications preoperatively was 2.6 (vs 1.7 in NST). A total of 165 (54.3\%) patients were on three or more topical medications at the time of listing for surgery (vs $9 \%$ in NST) and this was significantly greater than in the NST $(P<0.001)$. Figure 1 shows the distribution of the number of topical medications. A total of 48 patients (15.8\%) were on oral acetazolamide prior to surgery. A total of $56(18.4 \%)$ patients had undergone previous ophthalmic surgery involving the bulbar conjunctiva.

The reasons for undergoing trabeculectomy are outlined in Table 2. Advanced visual field loss was present in $64.5 \%$ of patients at the time of listing and this was significantly greater than in the NST $(50.5 \%)$ $(P<0.001)$.

The distribution of waiting times between listing and surgery is shown in Figure 2. A total of 284 (93.4\%) patients waited less than 3 months for trabeculectomy surgery and this was significantly more than the NST $(P<0.001)$.

\section{Type of surgery}

Intraoperative antimetabolites were applied in 147 $(48.4 \%)$ cases and its usage was significantly higher than in the NST $(6.4 \%)(P<0.01)$. 5FU was used in $61.9 \%$ of these cases and MMC in $38.1 \%$. Bleb intervention in the

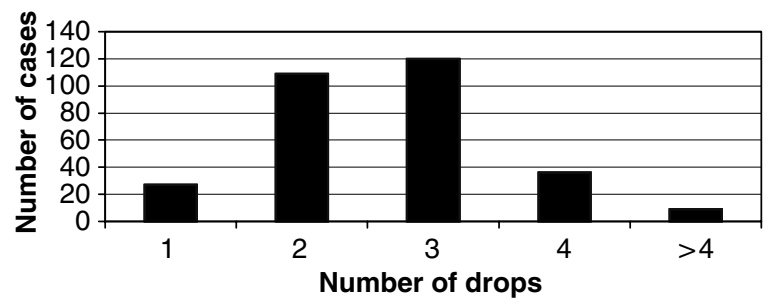

Figure 1 Number of topical antiglaucoma medications in the operated eye at the time of listing.

Table 2 Reason for listing for trabeculectomy

\begin{tabular}{lcc}
\hline Reason & $\begin{array}{c}\text { Number } \\
\text { of patients }\end{array}$ & $\begin{array}{c}\text { Percentage } \\
\text { of patients }\end{array}$ \\
\hline Poor IOP control with medication & 222 & 73.0 \\
Visual field deterioration & 186 & 61.2 \\
Progressive disc changes & 121 & 39.8 \\
Medication intolerance & 43 & 14.1 \\
Noncompliance with treatment & 22 & 7.2 \\
\hline
\end{tabular}

form of laser suture lysis, needling, or injection of 5FU was carried out in a total of $90(29.6 \%)$ cases and this was significantly more than in the NST $(P<0.001)$.

\section{Early complications}

Early complications occurred in the first two postoperative weeks in $164(53.9 \%)$ patients (vs 46.6\% patients in NST) and these are outlined in Table 3. The overall rate of early complications was significantly higher in the SESTS than in the NST $(P<0.025)$.

\section{Late complications}

In all, 33 patients (10.9\%) lost more than one line of Snellen visual acuity at 1 year (vs $18.8 \%$ patients in NST) and this was significantly less than in the NST $(P<0.01)$. The single and multiple reasons for this were cataract in $23(7.6 \%)$, visual field deterioration in nine $(3.0 \%)$, and other causes such as macula degeneration in $11(3.6 \%)$. Visual fields documented in $262(86.2 \%)$ patients 1 year post surgery showed deterioration in 60 patients $(19.7 \%)$. In 48 patients (15.8\%), this was due to glaucoma progression and in $10(3.3 \%)$ it was secondary to development of cataract. A total of 11 patients (3.6\%) had undergone cataract surgery at 1 year. Anti-glaucoma medication was instituted in nine $(3.0 \%)$ patients at the 1 year postoperative visit (vs $12.7 \%$ patients in NST)

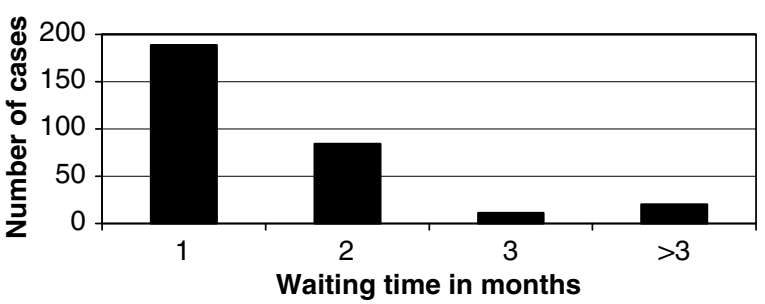

Figure 2 Waiting time: time interval between listing and trabeculectomy surgery.

Table 3 Early and late complication rates

\begin{tabular}{lcc}
\hline Complication & $\begin{array}{c}\text { Number } \\
\text { of patients }\end{array}$ & $\begin{array}{c}\text { Percentage } \\
\text { of patients }\end{array}$ \\
\hline $\begin{array}{l}\text { Early } \\
\quad \text { Shallow anterior chamber }\end{array}$ & 71 & \\
Hyphaema & 66 & 23.4 \\
Hypotony & 54 & 21.7 \\
Bleb leak & 50 & 17.8 \\
$\quad$ Choroidal detachment & 38 & 16.4 \\
Late & & 12.5 \\
$\quad$ 1 line Snellen vision loss & 33 & 10.9 \\
Visual field deterioration & 60 & 19.7 \\
Cataract surgery & 11 & 3.6 \\
\hline
\end{tabular}


and this was significantly less than in the NST $(P<0.001)$ No re-do trabeculectomies were performed during the time period studied.

\section{Outcome}

The mean IOP at 1 year following trabeculectomy was $14.1 \mathrm{mmHg}$ (95\% CI 13.6-14.6) (vs $14.4 \mathrm{mmHg}$ in NST) and this represents a mean reduction of $10.6 \mathrm{mmHg}$ from mean preoperative IOP at listing (95\% CI 9.6-11.4) (vs $11.8 \mathrm{mmHg}$ in NST). This equates to a mean \% reduction in IOP of $42.9 \%$ (vs $44.7 \%$ in NST). The mean IOP at the most recent outpatient visit (mean of 2.74 years postsurgery) was $13.9 \mathrm{mmHg}$ (95\% CI 13.4-14.3). Table 4 shows the outcome of trabeculectomy surgery at 1 year using the different criteria for success. There was no significant difference at IOP $<2 / 3$ listing IOP (qualified and unqualified) between the SESTS and the NST. However, significantly more patients achieved an unqualified success of IOP $<21 \mathrm{mmHg}(P<0.01)$ and an unqualified success of IOP $<16 \mathrm{mmHg}$ in the SESTS than the NST $(P<0.05)$. Table 5 shows the outcome of trabeculectomy according to the glaucoma subtypes.

\section{Glaucoma consultant vs higher surgical trainee}

Glaucoma consultants performed trabeculectomy surgery in 175 cases (57.6\%) and HST in 129 cases $(42.4 \%)$. Table 6 shows a comparison of outcomes between consultant and trainee. There was no significant difference in early complication rate between consultant and trainee. However, significantly trainee cases had to return more frequently to theatre $(P<0.025)$.

Furthermore, there was a greater degree of bleb intervention in trainee cases and this was also significant $(P<0.01)$. There was no significant difference in visual

Table 4 Outcome at 1 year post-trabeculectomy using different criteria for success

\begin{tabular}{lcc}
\hline Variable & Unqualified $\mathrm{n}(\%)$ & Qualified $\mathrm{n}(\%)$ \\
\hline IOP $<2 / 3$ listing IOP & $196(64.5 \%)$ & $199(65.5 \%)$ \\
IOP $<21 \mathrm{mmHg}$ & $277(91.1 \%)$ & $285(93.8 \%)$ \\
IOP $<16 \mathrm{mmHg}$ & $186(61.2 \%)$ & $191(62.8 \%)$ \\
\hline
\end{tabular}

loss of greater than one line of Snellen's visual acuity or visual field deterioration at 1 year between consultant and trainee. There was no significant difference in terms of successful outcome at 1 year (defined as IOP $<2 / 3$ listing IOP) between the glaucoma consultants $(68.0 \%$ of cases) and the trainees (62.0\% of cases).

\section{Discussion}

The aim of this survey was to achieve an accurate assessment of the outcome of patients undergoing trabeculectomy in South-East Scotland. Our outcome measures and complication rates compare favourably with the NST. There was no significant difference at IOP $<2 / 3$ listing IOP (qualified and unqualified) between the SESTS and NST. We feel that the evolution of powerful IOP-lowering drugs as well as the preoperative use of oral acetazolamide in $15.8 \%$ of our cohort has contributed to a lowering of listing IOP, which may explain this outcome.

The cohort of patients in our survey differed slightly from that of the NST where only patients with a diagnosis of POAG, NTG, PXF, or PDS were studied, whereas $18 \%$ of cases in our survey had complicated glaucoma such as chronic angle closure, iridocorneal endothelial syndrome, and neovascular and aphakic glaucoma. Furthermore, in the NST, patients were excluded if they had undergone any previous surgery involving the bulbar conjunctiva whereas we only excluded those patients who had a history of previous glaucoma surgery. These nonexclusion criteria together with the higher and longer use of preoperative glaucoma medication contribute to a higher risk of trabeculectomy failure than in the NST. It was also encouraging that IOP control was maintained at an average IOP of $13.9 \mathrm{mmHg}$ at a mean of 2.74 years following surgery.

The frequency of glaucoma surgery performed in Scotland has declined over the past decade by over $50 \%$. Figures from the Information and Statistics Division, which compiles Scottish National Health activity data, show that the number of glaucoma surgical procedures has fallen from 1756 in 1992 to 790 in 2003, representing a $55 \%$ fall. These data are similar to those reported by Whittaker et $a l^{5}$ for England from 1990 to 2000. They suggest several reasons for this trend, which include

Table 5 Outcome of trabeculectomy according to glaucoma subtypes

\begin{tabular}{lccccc}
\hline Diagnosis & POAG $(\mathrm{n}=199)$ & $P X F(\mathrm{n}=21)$ & NTG $(\mathrm{n}=23)$ & PDS $(\mathrm{n}=5)$ & Other $(\mathrm{n}=56)$ \\
\hline Mean IOP at listing (mmHg) & 23.8 & 26.8 & 17.8 & 20.0 & 29.3 \\
Mean IOP at 1 year (mmHg) & 14.1 & 13.5 & 11.8 & 13.4 & 15.1 \\
Success -IOP $<2 / 3$ listing IOP (\%) & 64.8 & 71.4 & 60.8 & 40.0 & 71.0 \\
\hline
\end{tabular}


Table 6 Comparison of outcomes between consultant and trainee

\begin{tabular}{lcc}
\hline & $\begin{array}{c}\text { Consultant } \\
(\mathrm{n}=175)\end{array}$ & $\begin{array}{c}\text { Trainee } \\
(\mathrm{n}=129)\end{array}$ \\
\hline Early complication & $91(52.0 \%)$ & $73(56.6 \%)$ \\
Return to theatre & $8(4.6 \%)$ & $20(15.5 \%)$ \\
Bleb intervention & $40(22.9 \%)$ & $50(38.8 \%)$ \\
$>1$ line Snellen vision loss & $23(13.1 \%)$ & $10(7.8 \%)$ \\
Visual field deterioration & $38(21.7 \%)$ & $22(17.1 \%)$ \\
Successful outcome & $119(68.0 \%)$ & $80(62.0 \%)$ \\
\hline
\end{tabular}

earlier presentation of glaucoma with less advanced disease, the introduction of new ocular hypotensive agents, and advances in surgical technique reducing the need for repeat procedures.

The decline in trabeculectomy surgery rates and the evolution of dedicated glaucoma clinics have resulted in the two glaucoma surgeons of South-East Scotland becoming responsible for performing $92 \%$ of trabeculectomies in the region, and they each either perform or supervise over a hundred glaucoma surgical procedures per annum. This trend is currently being experienced Scotland-wide, and individual unit figures show that between 70 and $80 \%$ of all glaucoma surgery performed in Scotland is now carried out by glaucoma specialists that make up $10 \%$ of the total Scottish consultant body. In contrast, during the NST period 91\% of UK consultant ophthalmologists performed glaucoma surgery.

Although at the time of the NST there was no statistical difference in successful outcome of trabeculectomy between glaucoma specialists and general (nonglaucoma) ophthalmologists, the odds of a successful outcome were a fifth higher for glaucoma specialists. ${ }^{2}$ A fall in trabeculectomy rates since the time of the NST has resulted in an even greater decline in the number of trabeculectomies being performed by general ophthalmologists. This has the potential to reduce the experience for general ophthalmologists in both performing a trabeculectomy and also, more importantly, the postoperative management of the failing trabeculectomy bleb.

It is conceivable that a similar survey to the NST, if performed, now would yield a statistical difference, with glaucoma consultants achieving more successful outcomes. If this were true, it would be difficult to justify the general ophthalmologist performing infrequent trabeculectomies and would provide a case for making glaucoma surgery the sole domain of the glaucoma specialist as suggested by Fraser. ${ }^{10}$ This would be another step towards the increased subspecialisation of ophthalmology in the UK not purely confined to glaucoma. ${ }^{11}$
Evidence from current trials suggests that lower IOPs correlate with visual field preservation and that the traditional target IOP of $21 \mathrm{mmHg}$ is obsolete. ${ }^{12}$ This has resulted in glaucoma specialists applying antimetabolites with increasing frequency with more bleb intervention to achieve lower IOPs. Antimetabolite usage in our audit was significantly higher than that of the NST, and this much higher rate of augmented trabeculectomy surgery is in keeping with other reports of a genuine increase in the use of antimetabolites. ${ }^{7}$ Siriwardena et al reported, in a national survey of antimetabolite usage in glaucoma, that $23 \%$ of consultants with a special interest in glaucoma used an antimetabolite in more than half their cases. Although antimetabolites have been shown to improve the success of trabeculectomy, their use is not without its hazards. Early complications have been reported to be more common with the use of antimetabolites. ${ }^{13}$ The overall rate of early complications was significantly higher in the SESTS than in the NST and this may be due to the increased use of antimetabolites. Reassuringly, other long-term studies indicate little correlation between early complications and long-term outcome. $^{14}$

In our survey, a comparison was made between the consultants and HST. Although it was not formally documented, in terms of patient selection the consultants tended to perform the more complicated cases (ie only eyes, advanced glaucoma, etc.). Our survey showed that patients had to return to theatre more frequently and had more postoperative bleb intervention when trainees performed surgery. Encouragingly however, it showed that trainees with close scrubbed supervision by the glaucoma consultant achieved similar rates of success in terms of the main outcome measures. This is important with regard to surgical training in an era of markedly increased public accountability that surgery performed by a trainee does not disadvantage patients. Although, as mentioned earlier in this discussion, future general ophthalmologists may not perform trabeculectomies, an exposure to glaucoma surgery in surgical training is important especially since the management of its complications may be necessary in an on-call capacity. The data from our survey show that there is no detriment to eventual outcome when trainees operate with glaucoma consultant supervision, and this can go some way to justify that trabeculectomy surgery remains an important aspect of surgical training.

In summary, our study highlights significant changes in the practice and outcome of trabeculectomy compared to the national audit conducted a decade ago. The consequences of these changes are the effect they may have on training issues as well as outcome of glaucoma surgery in the hands of general ophthalmologists. 


\section{References}

1 Edmunds B, Thompson JR, Salmon JF, Wormald RP. The national survey of trabeculectomy. I. Sample and methods. Eye 1999; 13: 524-530.

2 Edmunds B, Thompson JR, Salmon JF, Wormald RP. The national survey of trabeculectomy. II. Variations in operative technique and outcome. Eye 2001; 15: 441-448.

3 Edmunds B, Thompson JR, Salmon JF, Wormald RP. The national survey of trabeculectomy. III. Early and late complications. Eye 2002; 16: 297-303.

4 Bateman DN, Clark R, Azuara-Blanco A, Bain M, Forrest J. The impact of new drugs in the management of glaucoma in Scotland: observational study. BMJ 2001; 323: 1401-1402.

5 Whittaker KW, Gillow JT, Cunliffe IA. Is the role of trabeculectomy in glaucoma management changing? Eye 2001; 15: 449-452.

6 The Royal College of Ophthalmologists. The curriculum of higher specialist training in ophthalmology. 2003 (March), p 14, www.rcophth.ac.uk./edu_train/guides-curricular.html

7 Siriwardena D, Edmunds B, Wormald RPL, Khaw PT. National survey of antimetabolite use in glaucoma surgery in the United Kingdom. Br J Ophthalmol 2004; 88: 873-876.
8 Jay JL, Murdoch JR. The rate of visual field loss in untreated primary open angle glaucoma. Br J Ophthalmol 1993; 77(3): 176-178.

9 O'Brart DPS, Rowlands E, Islam N, Noury AMS. A randomised, prospective study comparing trabeculectomy augmented with antimetabolites with a viscocanalostomy technique for the management of open angle glaucoma uncontrolled by medical therapy. Br J Ophthalmol 2002; 86: 748-754.

10 Fraser S. Trabeculectomy and antimetabolites. $\mathrm{Br} \mathrm{J}$ Ophthalmol 2004; 88: 855-856.

11 Teenan DW, Sim KT, Hawksworth NR. Outcomes of corneal transplantation: a corneal surgeon $v$ s the general ophthalmologist. Eye 2003; 17: 727-730.

12 The Advanced Glaucoma Intervention Study (AGIS): 7. The relationship between control of intraocular pressure and visual field deterioration. The AGIS Investigators. Am J Ophthalmol 2000; 130(4): 429-440.

13 The Fluorouracil Filtering Surgery Study Group. Fluorouracil filtering surgery study one-year follow-up. Am J Ophthalmol 1989; 108(6): 625-635.

14 Watson PG, Jakeman C, Ozturk M, Barnett MF, Barnett F, Khaw KT. The complications of trabeculectomy (a 20-year follow-up). Eye 1990; 4(Part 3): 425-438. 